\title{
A terapia ocupacional na Estratégia de Saúde da Família - evidências de um estudo de caso no município de São Paulo
}

\section{The occupational therapy in the Health Family Strategy - evidence of a case study in the São Paulo city}

\author{
Tatiana de Andrade Jardim¹, Vanessa Cristina Afonso², \\ Isiélen Cardoso Pires ${ }^{3}$
}

\begin{abstract}
JARDIM, T. A. de; AFONSO, V. C.; PIRES, I. C. A terapia ocupacional na Estratégia de Saúde da Família - evidências de um estudo de caso no município de São Paulo. Rev. Ter. Ocup. Univ. São Paulo, v. 19, n. 3, p. 167-175, set./dez. 2008.

RESUMO: Destaca-se a atuação do terapeuta ocupacional junto ao Programa de Saúde da Família através das Equipes de Referência. Realizou-se uma pesquisa qualitativa, de caráter exploratório e corte transversal, através da aplicação de questionários com terapeutas ocupacionais do município de São Paulo, visando conhecer a realidade desse trabalho, suas características e confluência com os princípios e diretrizes do Sistema Único de Saúde (SUS) e, assim, promover o conhecimento e expansão desse serviço para a região noroeste do estado de São Paulo. Concluiu-se que o trabalho desenvolvido pelos profissionais condiz com os princípios e diretrizes da atenção básica no SUS, sua ação diretamente na comunidade proporciona busca ativa, identificação das necessidades dos locais de ação, ações de prevenção, promoção e reabilitação na própria comunidade, integrando serviço de saúde, equipe multiprofissional, comunidade e novos serviços de parcerias. Em contraponto, a estrutura do trabalho junto ao PSF apresenta alguns aspectos deficitários: falta de recursos humanos frente à demanda da comunidade e escassez de recursos materiais.
\end{abstract}

DESCRITORES: Terapia ocupacional. Saúde da família. Estratégias. Área de atuação profissional.

\footnotetext{
1. Terapeuta ocupacional. Mestre em Ciências da Reabilitação pela Faculdade de Medicina da USP. Colaboradora do Laboratório de Investigação e Intervenção em Saúde e Trabalho (FMUSP). Consultora em Reabilitação Profissional da Thymus Consultoria de Saúde Ltda.

2. Terapeuta ocupacional. Atua na área de Deficiência Mental como colaboradora na APAE de Fernandópolis e na área de Reabilitação Física.

3. Terapeuta ocupacional. Cursando especialização em Terapia Ocupacional na Faculdade de Medicina de São José do Rio Preto (Famerp).

Endereço para correspondência: Centro de Docência e Pesquisa em Terapia Ocupacional da FMUSP, Rua Cipotânea, 51 - Cidade Universitária, CEP: 05508-900, São Paulo-SP.
} 


\section{INTRODUÇÃO}

\section{Atenção básica em saúde no Brasil}

$\mathrm{A}$ Constituição Federal de 1988 instituiu uma nova proposta de organização e ação na área da saúde, o Sistema Único de Saúde (SUS), incorporando conceitos, princípios e uma nova lógica de gestão, que assume a saúde como um direito social universal, de caráter público e estabelece que a assistência deve ser oferecida por meio de uma rede regionalizada, hierarquizada e descentralizada, com foco único em cada esfera do governo sob o controle da sociedade (CHIORO; SCRAFF, 2005).

Para a implantação e efetivação do SUS destaca-se as Leis Orgânicas da Saúde (LOS), que regem sobre as condições para promoção, proteção e recuperação da saúde, não só como a ausência de doenças, mas sim como processo dinâmico de fatores presentes no cotidiano das pessoas (CHIORO; SCRAFF, 2005; GIL, 2006).

Os princípios e diretrizes do SUS são norteadores para o planejamento e implementação de todos os programas de ação na área da saúde, e em especial na atenção básica. É neste setor que se destaca a importância das ações na comunidade com participação social e enfoque preventivo e terapêutico, ao se considerar tais diretrizes chega-se ao principal programa vigente na atualidade na atenção básica: o Programa de Saúde da Família (CORNWALL; SHANKLAND, 2008).

No ano de 1991 criou-se o Programa de Agentes Comunitários da Saúde (PACS) composto por Agentes Comunitários de Saúde (ACS) que atuam traçando estratégias para uma ação diferenciada, realizada diretamente na comunidade, criando vínculos de co-responsabilidade com a população, com o intuito de desenvolver um trabalho preventivo, educativo e orientar as famílias da comunidade (BRASIL, 2000).

O PACS constituiu o processo de transição para a implantação do PSF. A partir deste trabalho executado pelos agentes comunitários, foi implantado em 1994, o Programa de Saúde da Família (BRASIL, 1997b; 2000).

O PSF constitui o primeiro nível da atenção à saúde do SUS, com ações programáticas e de demanda espontânea, de caráter individual e/ou coletivo, objetiva gerar promoção da saúde, prevenção de agravos, tratamento, reabilitação e manutenção da saúde, apresentando como principal desafio promover a qualidade de vida do indivíduo e a participação da comunidade no processo saúde-doença (BRASIL, 2006).
O Programa apresenta como diretrizes operacionais a integralidade e hierarquização, a territorialização e o cadastramento da clientela, a existência de equipe multiprofissional, a estimulação intersetorial, o estímulo à participação e controle social, a educação permanente dos profissionais das equipes de saúde da família e a adoção de instrumentos permanentes de acompanhamento e avaliação (BRASIL, 1997). É constituído por Equipe de Saúde da Família (ESF), considerada como principal fator na relação da comunidade com o PSF, sendo composta minimamente por um médico, um enfermeiro, auxiliar de enfermagem e seis agentes comunitários de saúde (CAPISTRANO FILHO, 1999).

Estas equipes são capacitadas de acordo com o Ministério da Saúde para

- Conhecer a realidade das famílias pelas quais é responsável, por meio de cadastramento e diagnóstico de suas características sociais, demográficas e epidemiológicas;

- Identificar os principais problemas de saúde e situações de risco aos quais a população está exposta;

- Elaborar, com a participação da comunidade, um plano local para enfrentar os determinantes do processo saúde/doença;

- Prestar assistência integral, na Unidade de Saúde da Família, na comunidade, no domicílio e no acompanhamento ao atendimento nos serviços de referência ambulatorial e hospitalar;

- Desenvolver ações educativas e intersetoriais para enfrentar os problemas de saúde identificados (BRASIL, 1997, p. 5).

Visando ampliar a abrangência e o escopo de ação na Atenção Básica, fortalecer o processo de territorialização e regionalização e ainda congruir com a Estratégia Saúde da Família criou-se, no ano de 2008, os Núcleos de Apoio a Saúde da Família (NASF) (BRASIL, 2008).

Os NASF podem ser classificados em NASF1 e NASF2, segundo Tabela 1. A Equipe de Referencia que compõe o NASF deve ser composta por um conjunto de profissionais de nível superior não possuindo nenhuma atividade parecida, podendo ser entre eles: Assistente Social, Professor de Educação Física, Farmacêutico, Fisioterapeuta, Fonoaudiólogo, Nutricionista, Psicóloga, Terapeuta Ocupacional e Médicos (em suas especialidades) (BRASIL, 2008). 
Tabela 1 - Regras para implantação de NASF1 e NASF2

\begin{tabular}{l|l|l|l}
\hline & Equipe mínima & Vínculo a __ equipes do PSF & Condições populacionais \\
\hline NASF1 & 5 profissionais & mínimo 8 e máximo 20 & inferior a 100.000 hab \\
NASF2 & 3 profissionais & mínimo 3 & superior a 100.000 hab. \\
\hline
\end{tabular}

Fonte: Brasil, 2008.

Dentre as referidas profissões, que compõe a equipe do NASF, este estudo enfatiza a atuação do Terapeuta Ocupacional junto a Estratégia de Saúde da Família.

\section{A Equipe de Referência}

A Equipe de Referência pode ser definida como,

um conjunto de profissionais considerados essenciais para a condução de problemas de saúde dentro de certo campo de conhecimento. Dentro desta lógica, a equipe de referencia é composta por distintos especialistas e profissionais encarregados de intervir sobre um mesmo objeto - problema de saúde - buscando atingir objetivos comuns e sendo responsáveis pela realização de um conjunto de tarefas, ainda que operando com diversos modos de intervenção (CAMPOS; DOMINITTI, 2007, p. 2).

Entende-se que as equipes de referência contribuem com ações de elaboração de projetos terapêuticos individualizados e com ações grupais, visando cuidar integralmente dos aspectos da saúde do indivíduo. Por se tratar de uma equipe multiprofissional permite enxergar diferentes caminhos para um projeto terapêutico, tendo sua atenção voltada exclusivamente para o bem estar e a qualidade de vida do usuário (BRASIL, 2004). Cada equipe de referência é responsável por território pré-determinado, fornecendo respaldo as ESF e sendo responsável pelas demandas desse local.

A origem do NASF pode ser remetida as demandas resultantes da realidade do trabalho das Equipes de Saúde da Família ao se depararem com sujeitos que apresentavam necessidades especiais na população de sua área de abrangência e a impossibilidade de atendimento dessa população. As ESF não possuem determinadas especialidades e sequer os agentes comunitários de saúde são profissionais da área da saúde, capacitados para lidar com tais demandas encontradas na comunidade.

Referente à realidade de atuação das ESF, o Ministério da Saúde evidencia que "as equipes se deparam, cotidianamente com problemas de 'Saúde Mental': 56\% das Equipes de
Saúde da Família referiram realizar alguma ação de saúde mental" (BRASIL, 2006, p.3). Evidencia-se, então, a importância e inexistência de profissionais especializados nas áreas de saúde mental e reabilitação física. Frente a realidade apresentada criou-se as Equipes de Saúde do Deficiente e Equipes de Saúde Mental, compostas por profissionais aptos em suas respectivas áreas.

Posteriormente, as Equipes de Referencia foram respaldadas por Equipes de Apoio Matricial, tidas como uma nova forma de arranjo organizacional, com a finalidade de aprimorar e ampliar a oferta de ações em saúde (BRASIL, 2004). Assim, as duas equipes juntas devem funcionar como meios indispensáveis para a humanização da atenção e da gestão em saúde. Lado a lado, promovem uma busca ativa na comunidade, criam vínculos que facilitam o atendimento, discutem os casos identificados, desenvolvem ações, programas e projetos terapêuticos.

\section{Terapia ocupacional e eixos de atuação}

A definição de Terapia Ocupacional, formulada pelo curso de terapia ocupacional da Universidade de São Paulo (USP) evidencia:

É um campo de conhecimento e de intervenção em saúde, educação e na esfera social, reunindo tecnologias orientadas para emancipação e autonomia das pessoas que, por razões ligadas a problemática específica, físicas, sensoriais, mentais, psicológicas e/ou sociais, apresentam, temporariamente ou definitivamente, dificuldade na inserção e participação na vida social. As intervenções em Terapia Ocupacional dimencionam-se pelo uso da atividade, elemento centralizador e orientador, na construção complexa e contextualizada do processo terapêutico (WFOT, 2003, p. 70).

A partir da definição acima e seguida da referida atuação do terapeuta ocupacional voltada para uma reabilitação baseada na comunidade, podemos entender que o profissional da área de Terapia Ocupacional é um elemento relevante em programas que atuam diretamente na comunidade, pois por sua formação interdisciplinar poderá estimular o desenvolvimento de ações que estejam 
focadas na esfera individual e na coletiva, contribuindo para o reconhecimento das necessidades da comunidade e dos indivíduos que nela residem. Há, então, uma possibilidade de atuação centrada e diretiva, porém sem desconsiderar os contextos nela envolvidos. Outro aspecto relevante é que os terapeutas ocupacionais atuam fornecendo respaldo também as famílias e a comunidade e não só aos indivíduos que necessitem diretamente do serviço (OLIVER; ALMEIDA, 2007).

Ademais, um fator que direciona o terapeuta ocupacional como um profissional habilitado para ter sua atuação baseada diretamente na comunidade é que ele, a partir de sua formação, estará preparado tanto para compreender, quanto para intervir nas dificuldades relacionadas a realização de atividades objetivas do cotidiano, como por exemplo podemos citar as Atividades de Vida Diária (AVDs) e as Atividades de Vida Prática (AVPs). Além disso, temos que considerar o conhecimento e possibilidade de contribuição desse profissional no que se refere a adaptações de ambiente, utensílios e meios de locomoção dos indivíduos que necessitem (NEISTADT, 2008).

Cabe ainda ressaltar que terapeutas ocupacionais desenvolvem atividades voltadas para a inserção e reinserção dos indivíduos na sociedade e no mercado de trabalho, o que possibilita a geração de renda para os indivíduos (OLIVER; ALMEIDA, 2007).

O estudo objetivou conhecer as características da organização do trabalho do terapeuta ocupacional na Estratégia Saúde da Família, sua inserção junto à equipe do Programa de Saúde da Família (PSF), as dificuldades e os aspectos prazerosos do trabalho do terapeuta ocupacional nesse contexto, suas possibilidades de desenvolver um trabalho multidisciplinar, além de, averiguar se a atuação do terapeuta ocupacional contribui para o fortalecimento da atenção básica em saúde no nosso país e se condiz com a organização e a proposta de ação do SUS.

\section{METODOLOGIA}

Trata-se de um estudo de caráter qualitativo e exploratório, com corte transversal e a utilização de questionário como instrumento. O questionário é uma técnica de investigação para coletar dados, com um número razoavelmente elevado de questões, sendo estas de qualidade impessoal e diretamente relacionadas com o tema em pauta, objetivando que os dados sejam obtidos de forma iniludível (CERVO; BERVIAN, 1996). Sua aplicação nos possibilita conhecimentos a partir da visão e/ou experiências dos próprios sujeitos pré-determinados.
O questionário aplicado foi formulado com questões qualitativas (em número de cinco) e quantitativas (em número de dezoito), somando assim um total de vinte e três questões, sendo as qualitativas objetivando proporcionar respostas livres, a fim de se conhecer aspectos subjetivos, opiniões e vivências desses profissionais. E questões quantitativas visando a obtenção de dados padrões relevantes, tais como o local dos atendimentos, o tipo de atendimento (grupal ou individual), a pertinência a uma equipe multidisciplinar, as demandas de pacientes e patologias e a origem dos recursos utilizados (pacientes ou serviços de saúde).

A pesquisa desenvolveu-se no ano no de 2008, sendo sujeitos da mesma quatro terapeutas ocupacionais que atuam na equipe de referência em Reabilitação Física - Saúde do Deficiente, junto ao PSF na Zona Norte do município de São Paulo.

$\mathrm{O}$ instrumento de pesquisa foi postado aos quatro participantes, sendo que anteriormente houve contato telefônico e por meio eletrônico para esclarecimentos e autorização de envio do instrumento. Este continha uma carta de apresentação com esclarecimentos, objetivos da pesquisa e contato dos pesquisadores, ficando acordado que o envio de resposta ao questionário seria caracterizado como consentimento em participar e autorização de uso das informações.

Os resultados foram obtidos a partir da avaliação dos questionários, comparação e discussão dos dados.

\section{RESULTADOS}

A população pesquisada é do sexo feminino, encontrase dentro da faixa etária de 21 a 36 anos, com média de 3 anos na função. A jornada de trabalho é de 30 a 40 horas semanais, sendo que o trabalho é realizado em UBS/USF, atendimentos domiciliares, locais cedidos pela comunidade. As modalidades de atendimento são individual e grupal, sendo que em média cada terapeuta coordena 3 grupos e o total de pacientes (atendimentos individuais e participantes de grupos) atendidos diariamente é em número de 13 por terapeuta, em média.

Os atendimentos são realizados individualmente e, quando necessário, através de uma equipe multidisciplinar, sendo esta composta pelos seguintes profissionais: terapeuta ocupacional, fonoaudiólogo, fisioterapeuta e equipe mínima do PSF.

Há prioridade de atendimento àqueles em situação de vulnerabilidade social, tendo como mais citados crianças, idosos e amputados recentes.

As maiores demandas de patologias encontradas em cada categoria são: 
JARDIM, T. A. de, et al. A terapia ocupacional na Estratégia. Rev. Ter. Ocup. Univ. São Paulo, v. 19, n. 3, p. 167-175, set./dez. 2008.

Ilustração 1 - Maiores demandas de patologias por categoria - Equipe de Saúde do Deficiente

\begin{tabular}{|c|l|}
\hline Categorias & \multicolumn{1}{c|}{ Patologias } \\
\hline Crianças & Atraso no Desenvolvimento Neuropsicomotor (ADNPM), TDA/H, encefalopatias e bebês de risco. \\
\hline Jovens & Seqüelas motoras, quadros neurológicos e ortopédicos e distúrbio de aprendizagem. \\
\hline Adultos & Acidente Vascular Encefálico (AVE) e amputados. \\
\hline Mulheres & Dores incapacitantes, quadros ortopédicos e quadros reumáticos. \\
\hline Idosos & AVE e senilidade. \\
\hline
\end{tabular}

Fonte: Questionários respondidos por terapeutas ocupacionais (2008).

Os materiais apresentados como mais utilizados foram papel, massa de modelar, lápis, canetas, brinquedos, jogos lúdicos, bola Bobath, elástico ou corda, colchonete ou tatame, andador, muletas, cadeira de rodas, equipamentos audiovisuais e educativos para grupos.

Estes materiais utilizados provêm do serviço de saúde e no caso de adaptações domiciliares utilizam-se, também, materiais do próprio paciente.

Relacionado à proposta de inserção do terapeuta ocupacional no PSF, foram citados os seguintes aspectos:

- atuam diretamente na prevenção da doença e agravos e promoção da saúde;

- mantém contato direto com a comunidade;

- proporciona atendimento a população que não tem outro tipo de acesso ao sistema de saúde;

- traz conhecimentos inovadores;

- oferece atendimento integral.

Em relação aos atendimentos em grupos, as atividades são voltadas para confecção e treino de adaptações, inserção do deficiente físico no trabalho e na sociedade, melhoria da qualidade de vida e integração.

No que diz a respeito à normatização que insere o terapeuta ocupacional no PSF, as terapeutas relatam que não interfere no cotidiano de trabalho, sendo que acreditam ser possível execução na prática dos princípios pensados para a Atenção Básica em saúde e para seus conseqüentes programas de ação e que fortalece para que o serviço se estenda à comunidade.

As maiores dificuldades apontadas foram a alta demanda de pacientes em contraponto à escassez de profissionais, a insuficiência de materiais e espaço adequado, o cansaço físico gerado nos profissionais e a carga horária de 20 ou 30 horas, incompatível a grande demanda que o projeto atende.
Os maiores prazeres citados como resultantes do trabalho que realizam, foram o estar junto aos pacientes e sua realidade, ser reconhecida na comunidade, ser procurada e identificada pela equipe do PSF e ter espaço e aceitação para interagir com a mesma, trabalhar em equipe e amadurecer como pessoa e profissionalmente.

Apresentaram como características benéficas para a população a busca ativa na comunidade, atuação dentro da realidade de cada população, acesso ao serviço de reabilitação e prevenção e a desmistificação do conceito de deficiência junto a comunidade e as ESF.

\section{DISCUSSÃO}

Os terapeutas ocupacionais inseridos na Estratégia de Saúde da Família não atuam somente em ambientes fechados restritos ao serviço de saúde, realizam ações na comunidade, considerando suas múltiplas potencialidades, como as residências dos sujeitos e, em especial, espaços comunitários e sociais, como praças, centros esportivos, salões comunitários, entre outros, ampliando o serviço de saúde para além de seus limites físicos institucionais. Consolida-se, assim, um eixo central da Estratégia Saúde da Família, o atuar diretamente na comunidade.

Quanto à modalidade de atendimentos prestados estes são realizados em grupo ou individualmente, a depender das necessidades e demandas da população, comunidade local. Nos atendimentos individuais tem-se atenção focada em um único paciente, objetivando a solução de suas demandas, enquanto que nos atendimentos grupais soma-se a essa ação a possibilidade do conviver, do se relacionar e a troca de vivencias e experiências entre o coletivo.

Refletindo sobre os atendimentos em grupo realizados pelas terapeutas ocupacionais temos que as atividades são 
voltadas para adaptações, para viabilizar, inserir e reinserir o indivíduo na sociedade e em seu trabalho, considerando que os atendimentos em grupo são um espaço onde "os participantes têm a possibilidade de experimentar outras formas de se relacionar e de vivenciar situações inéditas relativas ao fazer, possibilitando que a ação ganhe um sentido e um significado" (BALLARIN, 2007; p.40), assim, o grupo poderá ter como objetivo, por exemplo, a profissionalização ou a conscientização do auto-cuidado ou ainda o reestabelecimento da autonomia nas atividades de vida diária.

Ressaltando que para atingir seu sentido e seu significado é importante que os participantes estabeleçam uma relação entre a atividade proposta, o como fazer esta atividade e as intervenções do terapeuta ocupacional junto a este grupo, proporcionando um processo terapêutico com êxito (BALLARIN, 2007).

Quanto à inserção e/ou reinserção destes indivíduos que apresentem alguma deficiência, cabe citar algumas palavras de Rodrigues:

A inclusão da pessoa deficiente se dá no respeito às diferenças e às necessidades de cada um e não na tentativa de igualar todos institucionalmente. É um processo bidirecional, de construção coletiva que implica ajuste mútuo, cabendo à pessoa segregada a manifestação com relação às suas necessidades. A implementação dos ajustes e providencias necessárias que possibilitem o acesso imediato e definitivo à convivência em espaço comum caberá a sociedade (RODRIGUES, 2008; p.19).

A realização de grupos também permite aos profissionais a abrangência de um maior número de pacientes e de expansão de ações preventivas e/ou de orientação, capacitação.

Os grupos são organizados segundo as patologias, sendo que cada indivíduo vem com seu cuidador ou acompanhante e a terapeuta orienta e auxilia nas atividades dentro do grupo e capacita quanto à continuidade do trabalho de reabilitação ou prevenção fora do ambiente grupal, ou seja, para que se tenha desenvolvimento efetivo das atividades em outros espaços sociais, tais como, nas residências dos próprios sujeitos. Trata-se de uma possibilidade de preservar a qualidade do serviço e capacitar a comunidade, trabalhando no sentido de que também tenham responsabilidade no processo saúde-doença; outra diretriz relevante na Atenção Básica no SUS.

Os terapeutas ocupacionais atuam junto a uma equipe multidisciplinar, composta, entre outros, pela equipe mínima de saúde da família, fonoaudiólogo, fisioterapeuta, psicólogo, médico especialista, possibilitando, ou ainda, objetivando ao indivíduo da comunidade um atendimento mais completo. Uma equipe composta por profissionais de áreas complementares terá a possibilidade de realizar ações conjuntas, com atuações mais abrangentes e mais efetivas junto aos casos mais complexos ou anteriormente negligenciados.

Uma equipe se caracteriza pelo envolvimento de cada membro na tarefa e pelo comprometimento de todos nos resultados finais. As pessoas têm interação contínua, são interdependentes, compreendem-se mutuamente e participam das decisões (CAVALCANTI; GALVÃO, 2007, p. 35).

A atuação realizada diretamente na comunidade proporciona atendimento a população que não dispõe de outro tipo de acesso ao sistema de saúde, estando diretamente ligada a outro princípio de atenção em saúde no SUS, a "igualdade da assistência à saúde" (BRASIL, 1990; p.04), sem preconceitos ou privilégios de cada indivíduo.

Os recursos e materiais mais utilizados para os atendimentos das demandas em saúde do deficiente provêm do serviço de saúde e no caso de adaptações domiciliares, do paciente. A variabilidade de materiais que pode ser utilizado pelo terapeuta ocupacional é ampla e a determinação desses deve ser feita de acordo com os objetivos da ação terapêutica ocupacional e não a priori desta (HAGEDORN, 2007). Os materiais apresentados como mais utilizados para os atendimentos foram papel, massa de modelar, cola, tesoura, canetas, brinquedos, jogos lúdicos e materiais recicláveis com objetivo de estimulação global, reabilitação motora, estruturação de cotidiano e atividades expressivas. Há ainda os materiais específicos para ações que objetivam ganho de força muscular e auxílio para treino de marcha como, por exemplo, bolas bobath, andador, muletas e cadeira de rodas.

Dentre os aspectos subjetivos e opiniões das terapeutas no que se refere à proposta de inserção da Terapia Ocupacional no PSF, foram citados os seguintes aspectos: de acordo com o princípio da integralidade, um dos princípios estruturantes da atenção em saúde no SUS (BRASIL, 1990), a profissão de terapeuta ocupacional apresenta ações condizentes na medida em que atua diretamente na prevenção de doenças e agravos e promoção da saúde.

Outro princípio da atenção em saúde do SUS, que o profissional de Terapia Ocupacional também está fundamentado é o 'direito à informação' (BRASIL, 1990). Cabe aos terapeutas ocupacionais não só informar o próprio indivíduo sobre o que é sua patologia e o que pode ser feito para ajudá-lo e sim a todos da comunidade e até mesmo a ESF, trazendo conhecimentos inovadores, desmistificando o conceito de deficiência, surgindo um novo modo de olhar 
para o deficiente, oferecendo a população um atendimento integral.

O terapeuta ocupacional, junto a ESF irá trabalhar com a busca ativa em toda a comunidade de referencia, embasado em outro princípio da atenção a saúde do SUS, ‘participação da comunidade' (BRASIL, 1990). Atuará convivendo, participando das experiências cotidianas destes indivíduos, reconhecendo suas necessidades, considerando a realidade em que vivem tais sujeitos e seus familiares, enfim reconhecendo a comunidade da qual esses sujeitos fazem parte (OLIVER; ALMEIDA, 2007). Em outro aspecto, irá capacitar a comunidade para conhecer, discutir e buscar soluções para seus problemas locais e assumir seu papel no processo de co-gestão em saúde.

No que se refere a normatização que insere o terapeuta ocupacional junto a Estratégia Saúde da Família é interessante destacar que, segundo os resultados da pesquisa, o que é previsto para o trabalho da equipe no NASF não interfere nas ações e práticas no cotidiano de trabalho dos terapeutas ocupacionais, e sim, fornece respaldo no que diz respeito as ações de promoção, prevenção e reabilitação.

Quanto ao reconhecimento e as situações geradoras de satisfação no trabalho, relatam que apesar do desgaste devido a grande demanda, o projeto possibilita seu reconhecimento e aceitação na comunidade e dentro da equipe multidisciplinar. A devida efetivação do serviço, leva ao sucesso do programa, resultando em satisfação pessoal, que se torna primordial para a realização profissional das equipes.

Referem também a existência de dificuldades no cotidiano de trabalho, sendo elas, a alta demanda de atendimentos versus a escassez de profissionais, a falta de reposição de materiais e o contato com a miséria social existente na região.

As dificuldades citadas interferem no trabalho dos terapeutas ocupacionais, sendo que o contato com a realidade social é uma característica que permeia tal trabalho por ser esse uma proposta pautada na ação junto à comunidade, portanto, junto à sua realidade social, econômica, de saneamento básico, entre outros. A escassez de recursos humanos faz com que não consigam atender toda a demanda prontamente, gerando sensação de desconforto para a equipe. Já a falta de materiais está diretamente relacionada a realização de atividades na terapia ocupacional, pois o principal recurso de trabalho é a realização de atividades com objetivos terapêuticos. Através delas que as ações de avaliação, intervenção e o tratamento dos pacientes são fundamentadas na Terapia Ocupacional, buscando a melhora de todos as habilidades de desempenho destes indivíduos, estando principalmente voltadas para as atividades de vida diária (AVDs) e atividades de vida prática (AVPs) (NEISTADT, 2008). Acredita-se que as dificuldades enfrentadas pelos terapeutas ocupacionais condizem com algumas dificuldades vivenciadas pelos demais profissionais da ESF, conforme apresentado em outros estudos, sendo, portanto, uma interessante fonte de novas pesquisas no que tange ao trabalho prescrito na estratégia Saúde da Família (JARDIM; LANCMAN, 2009).

O trabalho é também composto de características positivas, reconhecidas nas ações consideradas benéficas para a população, tais como, busca ativa da demanda na comunidade, atuação na realidade do cotidiano desta mesma comunidade, tendo contado com a realidade de cada indivíduo, considerando as características e possibilidades deste; proporcionar novos conhecimentos sobre o portador de deficiência, mudando e ampliando a visão da sociedade para com estes indivíduos.

\section{CONCLUSÃO}

Ao se conhecer a proposta e a atuação de terapeutas ocupacionais na Equipe de Referência na zona Norte do Município de São Paulo, pode-se considerar que reflete um ganho qualitativo nas propostas de saúde diretamente para os serviços que compõem a atenção básica no SUS e para a população a ser atendida, uma vez que serviços de saúde com maior respaldo e abrangência em suas ações objetivam melhor qualidade de atendimento à população.

Concluiu-se que a atuação do terapeuta ocupacional junto ao PSF, apesar de um trabalho árduo e exaustivo, que encontra dificuldades, dentre as maiores podemos citar a grande demanda apresentada, a falta de materiais e recursos humanos e o conviver com diferenças sociais, pode ser considerado um trabalho gratificante devido ao reconhecimento gerado pela população e pela equipe de saúde onde atuam, ampliando seu espaço e o respeito pelo trabalho desenvolvido diariamente.

Concluiu-se, também, que a atuação do terapeuta ocupacional condiz com a organização, princípios e diretrizes da atenção básica no Sistema Único de Saúde, acrescentando e aprimorando os atendimentos voltados à comunidade.

A busca ativa que esse profissional realiza junto a ESF proporciona encontrar na comunidade indivíduos que estavam esquecidos, escondidos, sem acesso a tratamentos, provavelmente devido a escassez de conhecimento dos familiares, da própria comunidade frente a patologias, às questões econômicas e de acessibilidade.

Além de oferecer tratamento integral ao indivíduo, atuando dentro de uma equipe multidisciplinar, contribuindo 
JARDIM, T. A. de, et al. A terapia ocupacional na Estratégia. Rev. Ter. Ocup. Univ. São Paulo, v. 19, n. 3, p. 167-175, set./dez. 2008.

com ações e informações, o terapeuta ocupacional estará também orientando os familiares sobre o agir com este indivíduo, assim como a continuidade das atividades em casa, promovendo um tratamento mais intenso e eficaz.

JARDIM, T. A. de; AFONSO, V. C.; PIRES, I. C. The occupational therapy in the Health Family Strategy - evidence of a case study in the. São Paulo city. Rev. Ter. Ocup. Univ. São Paulo, v. 19, n. 3, p. 167-175, set./dez. 2008.

\begin{abstract}
The present research shown the occupational therapist's actuation in the Family Health Program, in the Local Reference team. There was a qualitative survey of exploratory nature and cross section through the application of questionnaires in occupational therapists in the municipality of São Paulo (Brasil), in order to know the reality of work, their characteristics and confluence with the principles and guidelines of the Health System (SUS) and thereby promote the expansion of knowledge and service to the northwestern region of São Paulo state. We concluded that the work developed by such professionals to agree with the principles and guidelines of Basic Attention in the SUS, its action directly in the community provides an active search, identification of the sites of action, actions for prevention, promotion and rehabilitation in the community, incorporating health service, team, community services and new partnerships. In contrast, the structure of the work with the PSF presents some loss: lack of human resources to fulfill the demand for the community and lack of material resources.
\end{abstract}

KEY WORDS: Occupational therapy. Family health. Strategies. Professional practice location.

\title{
REFERENCIAS
}

BRASIL. Ministério da Saúde. Legislação do SUS. Lei $n^{o}$. 8.080/90. Brasília/DF, 1990. Disponível em: http://dtr2004.saude. gov.br/dab/legislacao.php. Acesso em: 09 set. 2008.

BRASIL. Ministério da Saúde. A estratégia para a reorientação do modelo assistencial. Brasília, DF: Ministério da Saúde, 1997.

BRASIL, Ministério da Saúde. Secretaria de Assistência à Saúde. Programa de agentes comunitários de saúde. Brasília: Ministério da Saúde, 1997b.

BRASIL, Ministério da Saúde. Secretaria de Assistência à Saúde. Manual para organização da atenção básica em saúde. Brasília, DF, 1999. Disponível em: http://dtr2004.saude.gov.br/dab/ legislacao.php. Acesso em: 15 out. 2008.

BRASIL, Ministério da Saúde. Programa agentes comunitários de saúde - PACS. Brasília, DF: Ministério da Saúde; 2000.

BRASIL, Ministério da Saúde. Equipe de referência e apoio matricial. Brasília: Ministério da Saúde, 2004.

BRASIL, Ministério da Saúde. Secretaria de atenção à saúde, Departamento de Ações Programáticas e Estratégias/ Departamento de Atenção Básica. Saúde mental e atenção básica: o vínculo e o diálogo necessários. Brasília, DF: Ministério da Saúde, 2006.

BRASIL, Ministério da Saúde. Portaria $n^{\circ}$. 154 - Núcleo de apoio à saúde da família (NASF). Brasília, DF: Ministério da
Saúde, 2008.

CAMPOS, G. W. S.; DOMINITTI, A. C. Apoio matricial e equipe de referência: uma metodologia para gestão do trabalho interdisciplinar em saúde. Caderno de Saúde Pública, Rio de Janeiro, v.23/2, p. 399-407, fev. 2007.

CAPISTRANO FILHO, D. O programa saúde da família em São Paulo. Rev. Estudos Avançados (Dossiê Saúde Pública). São Paulo, v. 13/35, p. 89-100, 1999.

CAVALCANTI, A.; GALVÃO, C. Trabalho em equipe. In: Terapia ocupacional fundamentação e prática. Rio de Janeiro: Guanabara Koogan, 2007. p. 35-7.

CHIORO, A.; SCRAFF, A. A implantação do Sistema Único de Saúde. 2005. Disponível em: http://www.denem.org.br/2005/ arquivos/ok-112184655712.doc. Acesso em: 5 out. 2008.

CORNWALL, A.; SHANKLAND, A. Engaging citizens: lessons from building Brazil's national health system. Social Science Med., v. 66 , p. 2173-184, 2008.

GIL, C. R. R. Atenção primária, atenção básica e saúde da família: sinergias e singularidades do contexto brasileiro. Cad. Saude Publica, Rio de Janeiro, v. 22/6, p. 1171-81, jun. 2006.

HAGEDORN, R. Ferramentas para a prática em terapia ocupacional. São Paulo: Click Books, 2007. 
JARDIM, T. A. de, et al. A terapia ocupacional na Estratégia. Rev. Ter. Ocup. Univ. São Paulo, v. 19, n. 3, p. 167-175, set./dez. 2008.

JARDIM, T. A.; LANCMAN, S. Aspectos subjetivos do morar e trabalhar na mesma comunidade: a realidade vivenciada pelos agentes comunitários de saúde. Interface - Comunicação, Saúde e Educação, v. 13/28, p. 123-35, 2009.

NEISTADT, M. E. Introdução à avaliação e entrevista. In: WILLARD \& SPACKMAN. Terapia ocupacional. 9a. ed., São Paulo: Click Books, 2008.

OLIVER, F. C.; ALMEIDA, M. C. Reabilitação baseada na comu- nidade. In: CAVALCANTI, A.; GALVÃO, C. Terapia ocupacional fundamentação e prática. Rio de Janeiro: Guanabara Koogan, 2007. p. 125-132.

RODRIGUES, A. C. Reabilitação, práticas inclusivas e estratégias para a ação. São Paulo: Andreoli, 2008.

WORLD FEDERATION OF OCCUPATIONAL THERAPISTS (WFOT). Definições de terapia ocupacional. Apoio: Faculdades Salesianas de Lins, CETO/ SP, ABRATO; 2003. 